\title{
Effects of Dietary Intervention on Gut Microbiota and Metabolic-Nutritional Profile of Outpatients with Non-Alcoholic Steatohepatitis: a Randomized Clinical Trial
}

\author{
Fabiana de Faria Ghetti ${ }^{1}$, Daiane Gonçalves Oliveira ${ }^{1}$, Juliano Machado de Oliveira ${ }^{1}$, Lincoln Eduardo Villela Vieira de \\ Castro Ferreira ${ }^{1}$, Dionéia Evangelista Cesar ${ }^{2}$, Ana Paula Boroni Moreira ${ }^{3}$
}

1) Universitary Hospital and

School of Medicine, Federal University of Juiz de Fora 2) Biology Departament, Federal University of Juiz de Fora

3) Nutrition Department, Federal University of Juiz de Fora, Minas Gerais, Brasil

\footnotetext{
Address for correspondence: Fabiana de Faria Ghetti Universidade Federal de Juiz de Fora

Departamento de Nutrição. Bairro Martelos, s/n, CEP 36036-330, Juiz de Fora, Minas Gerais, Brazil bia.ghetti@hotmail.com
}

Received: 17.06.2019 Accepted: 27.08.2019

\section{ABSTRACT}

Background \& Aims: Modulation of the gut microbiota emerges as a therapeutic possibility to improve health. Our objective was to compare the impact of three months of intervention with diet plus nutritional orientation versus only nutritional orientation on the gut microbiota and metabolic-nutritional profile of outpatients with non-alcoholic steatohepatitis.

Methods: It was a randomized clinical trial with 40 outpatients ( $49.48 \pm 10.3$ years), allocated in two groups: DIET group ( $\mathrm{n}=20)$, who received diet $(1.651 .34 \pm 263.25 \mathrm{kcal} ; 47 \%$ carbohydrates, $28 \%$ lipids, $25 \%$ proteins, $30 \mathrm{~g}$ fibers) and nutritional orientation, and control group $(\mathrm{n}=20)$, which received only nutritional orientation. Results: The DIET group, in relation to baseline, presented a reduction in body weight $(\mathrm{p}<0.001), \mathrm{BMI}$ $(p<0.001)$, waist circumference $(p=0.001)$, percentage of fat $(p=0.002)$, serum aspartate aminotransferase $(\mathrm{p}<0.001)$, alanine aminotransferase $(\mathrm{p}<0.001), \gamma$-glutamyltransferase $(\mathrm{p}=0.001)$, glycemia $(\mathrm{p}=0.003)$, homeostasis model assessment of insulin resistance $(\mathrm{p}=0.017)$, total cholesterol $(\mathrm{p}=0.014)$, and triacylglycerols $(\mathrm{p}=0.008)$, whereas the control group did not present changes. After intervention, the small intestinal bacterial overgrowth frequency was $30 \%$ in the DIET group and $45 \%$ in the control group ( $\mathrm{p}=0.327)$. In the DIET group, an increase in the density of total microorganisms $\left(3.76 \pm 7.17 \times 10^{8}\right.$ cells $\left.^{-1} ; \mathrm{p}=0.048\right)$ was detected, while in the control group reduced Bacteroidetes $\left(-0.77 \pm 2.01 \times 10^{8}\right.$ cells g $\left.^{-1}, \mathrm{p}=0.044\right)$ and Verrucomicrobiales $(-0.46$ $\pm 0.75 \times 10^{8}$ cells $\left.\mathrm{g}^{-1} ; \mathrm{p}=0.022\right)$ were observed.

Conclusions: The results suggest that exclusively dietary modifications contribute to health promotion in non-alcoholic steatohepatitis and should be the basis of nutritional treatment for this condition.

Key words: non-alcoholic fatty liver disease - fatty liver - non-alcoholic steatohepatitis - microbiota -dysbiosis.

Abbreviations: FISH: fluorescence in situ hybridization; LPS: lipopolysaccharide; NAFLD: non-alcoholic fatty liver disease; NAS: NAFLD activity score; NASH: non-alcoholic steatohepatitis; SIBO: small intestinal bacterial overgrowth; TTE: total energy expenditure.

\section{INTRODUCTION}

Non-alcoholic steatohepatitis (NASH) is defined as the presence of a least $5 \%$ hepatic steatosis and inflammation with hepatocyte injury (e.g., ballooning), with or without fibrosis [1]. While the prevalence of NASH ranges from $2 \%$ to $5 \%$ in the general population $[2,3]$, approximately $70 \%$ of obese individuals are affected by this condition [4] Although in most cases it does not cause symptoms, NASH increases the risk of cirrhosis, hepatic insufficiency and hepatocellular carcinoma [4-6].

The NASH cause is still unclear, but studies have suggested the role of the gut microbiota in the pathogenesis of this disease $[7,8]$. Changes in the gut microbiota (called "dysbiosis") have consequences on energetic homeostasis, resulting in obesity and hepatic steatosis [9]. Dysbiosis is also responsible for increased intestinal permeability and circulating lipopolysaccharide (LPS) concentration, causing metabolic endotoxemia [10]. Moreover, dysbiosis can alter the metabolism of choline [11] and bile acids [12] in NASH and increase endogenous ethanol production [13]. However, few studies have evaluated the gut microbiota in NASH patients. Some findings suggest that NASH patients present a higher prevalence of small intestinal bacterial overgrowth (SIBO) 
$[14,15]$. In addition, the composition of fecal microbiota is different in this population when compared to healthy individuals $[16,17]$.

There is evidence that the amount of energy and the proportion of the three macronutrients in the diet have the potential to modulate the gut microbiota [18]. However, to date, no study has investigated the effects of exclusively dietary modification on the gut microbiota of NASH outpatients. Thus, the objective of this study was to evaluate the effects of three months of dietary intervention in the gut microbiota and metabolic-nutritional profile of NASH outpatients.

\section{METHODS}

\section{Population and experimental design}

Research outpatients were recruited from the Hepatology Service of the University Hospital at the Federal University of Juiz de Fora, Brazil. Outpatients were included from July 2015 to September 2017, and the inclusion criteria were: age $\geq 18$ years, of both genders and previous diagnosis of NASH. The NASH diagnosis was based on clinical and laboratory profiles and liver biopsies, which were routine evaluations in the Hepatology Service, as previously described by De Oliveira et al. [19].

The criteria for non-inclusion were: presence of other relevant liver diseases diagnosed through laboratory tests for the following diseases: chronic hepatitis $\mathrm{B}$, chronic hepatitis C, autoimmune hepatitis (anti-smooth muscle antibody, anti-mitochondrial antibody, and antinuclear antibody) and hemochromatosis (ferritin). In addition, HIV-infection, drug- induced hepatic disease, excessive alcohol consumption ( $>30$ $\mathrm{g}$ /day in men or $>20 \mathrm{~g} /$ day in women) and antibiotics in the two months prior to study enrollment represented exclusion criteria. According to the sample calculation [20], using a power of $90 \%$ and significance level of $5 \%$ to detect a difference of $46.5 \%$ in the prevalence of SIBO [15], 19 outpatients had to be recruited in each group. Initially, 45 outpatients met the inclusion criteria; however, 1 outpatient declined to participate and 4 outpatients ( 2 in each group) were excluded because they did not complete the three-month experimental protocol. Therefore, 40 outpatients who completed the experimental protocol were included in the analysis (Fig. 1).

This randomized, open-label clinical trial was carried on for three months. Outpatients were randomized into two groups: the experimental group, which received individualized diet plus nutritional orientation (DIET group, $n=20$ ), and control group, which received only nutritional orientation (CT group, $n=20$ ). A researcher who was not directly involved in the sample selection was responsible for the computer block randomization. For each four outpatients who agreed to participate in the study, two were drawn to belong to the DIET group, and the other two were drawn for the CT group, and so on, until the estimated sample number was reached. Block randomization was used to avoid or lessen possible imbalances at some point in the randomization process. The distribution of the variables age and gender of the outpatients was similar for the DIET and CT groups, confirming the appropriateness of the randomization process at the beginning of the study. At baseline, the outpatients were evaluated in relation to the variables of interest. Outpatients from both groups were

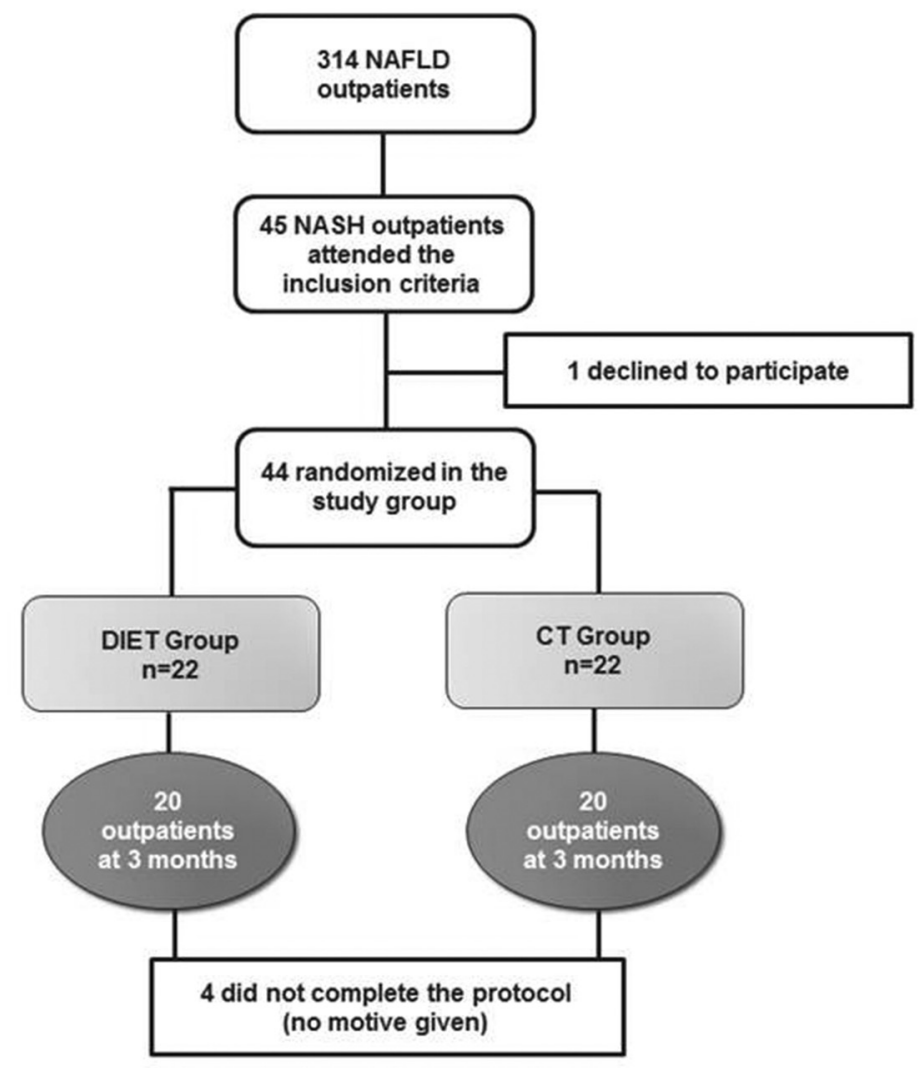

Fig. 1. Recruitment flow chart and study design. NASH, non-alcoholic steatohepatitis; NAFLD, non-alcoholic fatty liver disease. 
followed up in individual consultations, monthly, by the same nutritionist. All volunteers were instructed to maintain the same level of physical activity during the trial period. After the intervention period, the groups were submitted to the same evaluations (Fig. 1).

This study was conducted in accordance with the guidelines established in the Declaration of Helsinki and all procedures involving human subjects were approved by the Human Research Ethics Committee of the University Hospital at the Federal University of Juiz de Fora, Brazil (protocol number $1.129 .516 / 2015)$. All subjects provided written informed consent. This study is part of a large project entitled "Effects of nutritional intervention on the nutritional profile, inflammation and intestinal health of outpatients with liver disease", registered at http://ensaiosclinicos.gov.br/rg/RBR2 rcph2/, with the general objective to evaluate nutritional status, inflammatory parameters and intestinal health of outpatients with liver cirrhosis and NAFLD before and after nutritional intervention. Specifically in this study, we intended to evaluate the effects of three months of dietary intervention in the gut microbiota and metabolic-nutritional profile of NASH outpatients.

\section{Demographic data}

The anamnesis consisted of questions regarding personal data, clinical history, and level of physical activity according to the International Physical Activity Questionnaire (IPAQ) short version [21]. The diagnosis of systemic arterial hypertension and type 2 diabetes was obtained from medical records. The presence of metabolic syndrome was defined according to the criteria of the International Diabetes Federation [22].

\section{Histology of the liver}

The liver biopsy specimens, collected before inclusion in the study, were reviewed by a blind pathologist for the clinical characteristics of the outpatients. The histological analysis was based on the Non-alcoholic Fatty Liver Disease (NAFLD) activity score (NAS), developed by the Nonalcoholic Steatohepatitis Clinical Research Network [23].

\section{Biochemical evaluation}

After $12 \mathrm{~h}$ of fasting, venous samples were taken for analysis of liver biochemistry: alanine aminotransferase (ALT), aspartate aminotransferase (AST), gammaglutamiltransferase (GGT), total bilirubin and metabolic parameters (lipid profile and fasting insulin and glucose). The blood was separated by centrifugation and immediately analyzed in the Laboratory of Clinical Analysis of the University Hospital of the Federal University of Juiz de Fora. AST, ALT, GGT, total bilirubin, fasting glucose, total cholesterol, high density lipoprotein (HDL) and triacylglycerols (TAG) were analyzed by standard laboratory methods using a self-analyzer WIENER LAB, CT600i. Fasting insulin was determined by a self-analyzer Architect, i1000SR. Low density lipoprotein (LDL) was calculated according to Friedewald et al. [24]. The evaluation of the homeostasis model of insulin resistance (HOMA-IR) was calculated according to Matthews et al. [25].

\section{Anthropometric and dietary assessment}

Weight, height, waist circumference, and body composition were assessed in the fasting state. Waist circumference was measured at the midpoint between the iliac crest and the last rib. Body mass index (BMI) was calculated [26]. Body fat was measured by bioimpedance analyzer (RJL Systems, Inc (EUA) - Model Quantum II, serial number Q4631IID) as described previously [27].

Dietary assessment was performed through the Quantitative Food Frequency Questionnaire, validated for the Brazilian population [28]. All dietary questionnaires were analyzed by the same nutritionist and the daily intake of energy and nutrients (carbohydrates, fiber, lipids, saturated and unsaturated fatty acids and proteins) were calculated using Dietpro 5i software (Agromídia, Viçosa, Brazil), based on composition proposed by TACO (2004) and USDA (2003).

\section{Characterization of fecal microbiota}

Samples of feces were collected by outpatients in sterile vials and kept refrigerated for up to 24 hours until they were taken to the laboratory. Fecal samples were processed for microbiological analysis by fluorescence in situ hybridization (FISH) (Supplementary Table I). The samples were fixed with paraformaldehyde (final concentration $2 \%$ ), and a $0.01 \%$ Tween solution was added to each sample. The samples were then sonicated (Vibra Cell VCX 130PB, Sonics \& Materials ${ }^{\circledR}$ ) three times (range $110.7 \mu \mathrm{m}$ for $60 \mathrm{~s}$ ). After sonication, the samples were centrifuged at $500 \mathrm{~g}$ for $5 \mathrm{~min}$. The supernatant was removed and the remaining contents was washed twice with ultrapure water. The three supernatant fractions were placed in one vial and shaken vigorously. Aliquots of each sample were diluted (100x) and filtered through polycarbonate filters (Nuclepore ${ }^{\varpi}-0.2 \mu \mathrm{m}$ ) and stored in a refrigerator until the hybridization process.

Subsequently, the samples were submitted to FISH protocol [29] for identification and quantification of microbial groups. Thus, 24 oligonucleotide probes labeled with $\mathrm{Cy} 3$ fluorochrome were used. A negative control probe (5'CCTAGTAGACGCCGTCGAC-3'), which has no specificity for any bacterial group, was also used to evaluate the efficiency of the hybridization. The density of the microorganisms ( $\mathrm{x}$ $10^{8}$ cells $\left.\mathrm{g}^{-1}\right)$ was determined by direct counting at $100 \times$ magnification using an epifluorescence microscope (Olympus BX-60) equipped with the U-N41007, U-MWU2, U-MWB2 and U-MWG2 optical filters.

\section{Small intestinal bacterial overgrowth assessment}

The outpatients were submitted to expired hydrogen breath test $\left(\mathrm{H}_{2}\right)$ and methane $\left(\mathrm{CH}_{4}\right)$ for the detection of SIBO. For the test, outpatients received preparation guidelines, which included: not using antibiotics in the last 4 weeks prior to the test, discontinuing the use of proton pump inhibitors one week prior to the test, and avoiding fiber-rich foods the day before. Tests were performed on QuinTron BreathTracker ${ }^{\mathrm{Tm}}$ Digital MicroLyzer, with the outpatient having fasted for 10 hours. On the day of examination, expired alveolar air was collected in specific collection bags prior to ingestion of the test substrate $(15 \mathrm{ml}$ syrup at the concentration of $667 \mathrm{mg}$ of lactulose, followed by the consumption of $200 \mathrm{ml}$ of filtered 
water) at time $0^{\prime}$ (fasting), and after the use of lactulose at times 15, 30, 45, 60', 90 , and 120'. Elevations of $\mathrm{H}_{2} \geq 20$ ppm and/or $\mathrm{CH}_{4} \geq 10 \mathrm{ppm}$ were considered positive for SIBO from the 0 ' to 90 ' time dosing [30].

\section{Dietary intervention}

The dietary intervention consisted of the prescription of individualized diet plus nutritional orientation (DIET group) or only of nutritional orientation-control group (CT group). The clinical history, nutritional status, lifestyle, eating habits and the calculation of the nutritional needs of each patient were considered for the preparation of the individualized diet. The total energy expenditure (TEE) was calculated according to the Dietary Reference Intakes (DRIs) [31]. The average TEE of the DIET and CT groups were $2276.3 \pm 358.1 \mathrm{kcal}$ and $2269.9 \pm$ $506.5 \mathrm{kcal}$ per day $(\mathrm{p}=0.963)$, respectively. Overweight or obese outpatients received a hypocaloric diet, with a deficit of 500 to $750 \mathrm{kcal}$ per day (mean of $624.9 \pm 94.8 \mathrm{kcal}$ ) in relation to TEE [32]. The caloric distribution of macronutrients and the supply of dietary fiber were based on previous recommendations for fatty liver disease [33]. The characteristics of the diet prescribed to the DIET group are described in Table I. The nutritional orientations were based on the recommendations of the food guide for the Brazilian population [34].

Table I. Characteristics of the diet prescribed to the DIET group

\begin{tabular}{|c|c|c|c|c|c|}
\hline & & \multicolumn{2}{|c|}{ Grams } & \multicolumn{2}{|c|}{$\%$ TEE } \\
\hline & & Mean & SE & Mean & SE \\
\hline \multicolumn{6}{|l|}{ Energy (kcal) } \\
\hline Mean & $1,651.3$ & & & & \\
\hline SEM & 58.8 & & & & \\
\hline Carbohydrates & & 198.4 & 7.3 & 47.4 & 0.8 \\
\hline Lipids & & 51.5 & 2.4 & 27.7 & 0.7 \\
\hline Protein & & 103.9 & 4.2 & 24.9 & 0.7 \\
\hline SFA & & 12.6 & 0.8 & 6.9 & 0.4 \\
\hline PUFA & & 14.9 & 0.6 & 8.1 & 0.4 \\
\hline MUFA & & 19.3 & 0.6 & 10.6 & 0.3 \\
\hline Fibers & & 30.3 & 1.06 & & \\
\hline
\end{tabular}

Data are represented as mean and standard error. SFA: saturated fatty acid; PUFA: polyunsaturated fatty acid; MUFA: monounsaturated fatty acid; TEE: total energy expenditure

\section{Statistical analysis}

Statistical analysis was performed using SPSS ${ }^{\circledast}$ software (version 20.0, SPSS Inc., United States of America). Parametric and non-parametric tests were used according to the normality tests (Shapiro-Wilk) and homogeneity of variances (Levene). A significance level of $5 \%$ was adopted $(\mathrm{p}<0.05)$. Continuous variables were represented by mean and standard error of the mean. Categorical variables were expressed as absolute (n) and relative (\%) frequencies. The $t$-test was used for independent samples or Mann-Whitney $U$ test for comparing continuous variables at baseline and after intervention between groups. In order to compare the continuous variables before and after intervention, the $t$-test for paired samples or Wilcoxon test was used. Categorical variables were compared by the chisquare test $\left(\chi^{2}\right)$ or Fisher's Exact test (between groups) and the
McNemar test (within the group). The analysis "per protocol" has been chosen and not "intention-to-treat".

\section{RESULTS}

Of the 40 outpatients included in the study, the mean age was $49.4 \pm 2.3$ years (ranging from 25 to 69 years) and $52.5 \%$ $(\mathrm{n}=21)$ were males. At baseline, DIET and CT groups were similar in relation to demographic characteristics, clinical history, histological analysis (Table II), food consumption (Table III), physical activity level and metabolic-nutritional profile (Table IV), reinforcing homogeneity of the groups at baseline and the appropriateness of the randomization process.

After three months of dietary intervention, the DIET group presented a reduction in most of the metabolic and nutritional parameters, whereas the CT group did not present changes (Table IV). In the DIET group, body weight decreased by $4.7 \%$ (about $4 \mathrm{~kg}$ ). BMI, waist circumference and percentage of body fat decreased by $3.7 \%, 3.6 \%$ and $7.1 \%$, respectively, in relation to baseline measurements. In addition, GGT, glycemia and TAG values normalized after dietary intervention, and total cholesterol decreased by $6.8 \%$. After three months of follow-up, BMI, HOMA-IR and insulin, AST and GGT levels were significantly lower in the DIET group, compared to CT group. At the end of the study, the volunteers had not changed their level of physical activity, and there was no difference in the IPAQ classification between the groups.

Table II. Demographic characteristics, clinical history and histological analysis of patients with non-alcoholic steatohepatitis before inclusion in the study

\begin{tabular}{|c|c|c|c|c|c|c|}
\hline & \multicolumn{3}{|c|}{ DIET $(n=20)$} & \multicolumn{2}{|c|}{$\mathrm{CT}(\mathrm{n}=20)$} & \multirow[t]{2}{*}{$\mathrm{p}$} \\
\hline & $\%$ & & $\mathrm{n}$ & $\%$ & $\mathrm{n}$ & \\
\hline Age (years) & & & & & & 0.48 \\
\hline Mean & & 48.3 & & & & \\
\hline $\mathrm{SE}$ & & 2.3 & & & & \\
\hline Gender (male) & 60 & & 12 & 45 & 9 & 0.34 \\
\hline SAH (yes) & 35 & & 7 & 50 & 10 & 0.33 \\
\hline T2D (yes) & 10 & & 2 & 30 & 6 & 0.23 \\
\hline Steatosis & & & & & & 0.91 \\
\hline $5 \%-33 \%$ & 15 & & 3 & 15 & 3 & \\
\hline $34 \%-66 \%$ & 40 & & 8 & 50 & 10 & \\
\hline$>66 \%$ & 45 & & 9 & 35 & 7 & \\
\hline $\begin{array}{l}\text { Lobular } \\
\text { inflammation }\end{array}$ & & & & & & 1.00 \\
\hline$<2$ foci/200x & 75 & & 15 & 75 & 15 & \\
\hline 2 a 4 foci/ $200 x$ & 25 & & 5 & 25 & 5 & \\
\hline Ballooning & & & & & & 0.20 \\
\hline Few cells & 55 & & 11 & 35 & 7 & \\
\hline Many cells & 45 & & 9 & 65 & 13 & \\
\hline Fibrosis stage & & & & & & 0.38 \\
\hline F0 & 55 & & 11 & 30 & 6 & \\
\hline $\mathrm{F} 1$ & 40 & & 8 & 55 & 11 & \\
\hline $\mathrm{F} 2$ & 5 & & 1 & 10 & 2 & \\
\hline F3 & 0 & & 0 & 5 & 1 & \\
\hline
\end{tabular}

T2D: type 2 diabetes; SD: standard deviation; SAH: systemic arterial hypertension; NAS: non-alcoholic fatty liver disease activity score; SE: standard error 
Table III. Dietary intake of patients with non-alcoholic steatohepatitis at baseline

\begin{tabular}{cccccc}
\hline & \multicolumn{2}{c}{ DIET $(\mathrm{n}=20)$} & \multicolumn{2}{c}{ CT $(\mathrm{n}=20)$} \\
\hline & Mean & SE & Mean & SE & P \\
\hline Energy (kcal) & $2,444.0$ & 142.4 & $2,244.8$ & 142.2 & 0.33 \\
Carbohydrates & & & & & \\
g & 310.5 & 22.9 & 297.9 & 20.4 & 0.70 \\
\% & 50.3 & 1.6 & 52.3 & 1.5 & 0.38 \\
Lipids & & & & & \\
g & 87.1 & 6.7 & 78.7 & 7.3 & 0.33 \\
\% & 31.8 & 1.3 & 29.6 & 1.2 & 0.23 \\
Proteins & & & & & \\
g & 104.8 & 7.0 & 92.1 & 5.3 & 0.15 \\
\% & 17.8 & 1.2 & 17.7 & 1.1 & 0.93 \\
Fibers (g) & 17.8 & 1.2 & 18.9 & 1.3 & 0.53 \\
SFA & & & & & \\
g & 19.4 & 2.4 & 16.8 & 2.9 & 0.19 \\
\% & 7.0 & 0.7 & 6.4 & 0.9 & 0.35 \\
PUFA & & & & & \\
g & 26.7 & 1.8 & 22.0 & 2.4 & 0.13 \\
\% & 9.9 & 0.5 & 8.7 & 0.6 & 0.15 \\
MUFA & & & & & \\
g & 21.9 & 2.5 & 15.8 & 1.8 & 0.07 \\
$\%$ & 8.0 & 0.7 & 6.1 & 0.5 & 0.15 \\
\hline
\end{tabular}

SFA: saturated fatty acid; PUFA: polyunsaturated fatty acid; MUFA: monounsaturated fatty acid; SE: standard error.

At baseline, the DIET and CT groups were similar in relation to SIBO frequency ( $30 \%$ vs. $25 \%$, respectively, $\mathrm{p}=0.723$ ). After three months of dietary intervention, there was no significant change in the proportion of positive tests within the DIET group (30\%, $\mathrm{p}=1.000)$, within the CT group $(45 \%, p=0.289)$ and between groups $(\mathrm{p}=0.327)$. Regarding the composition of the fecal microbiota, there was no significant difference between the groups at baseline and at 3 months (Fig. 2 and 3 ). However, after the intervention, the density of total microorganisms increased in the DIET group in relation to the basal one (Fig. 2), while in the CT group density decreased for Bacteroidetes $(\mathrm{p}=0.04)$ and Verrucomicrobiales $(\mathrm{p}=0.02)$ (Fig. 3 ). In addition, it was observed that outpatients who received nutritional orientation only (CT group) had a tendency to decrease Actinobacteria ( $\mathrm{p}=0.05)$ (Fig. 3 ) and tendency to increase Escherichia coli ( $\mathrm{p}=0.05)$ density (Fig. 4).

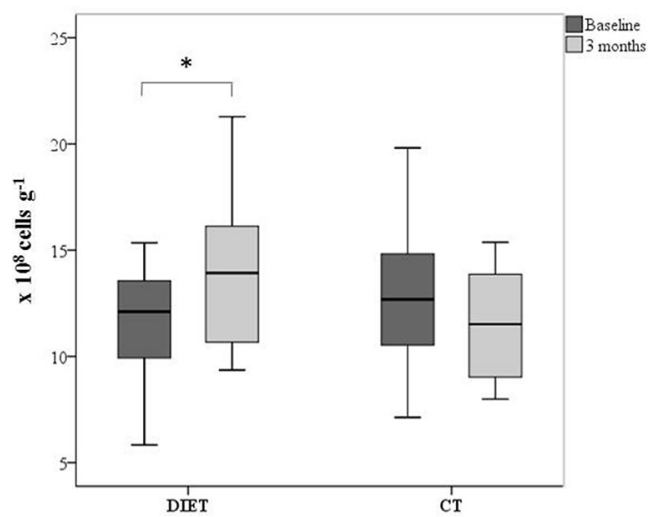

Fig. 2. Density of total microorganisms in patients with non-alcoholic steatohepatitis at baseline and 3 months, according to the study group. ${ }^{*}$ Significant difference between baseline and 3 months $(\mathrm{p}=0.04)$ in the DIET group, using Wilcoxon test.

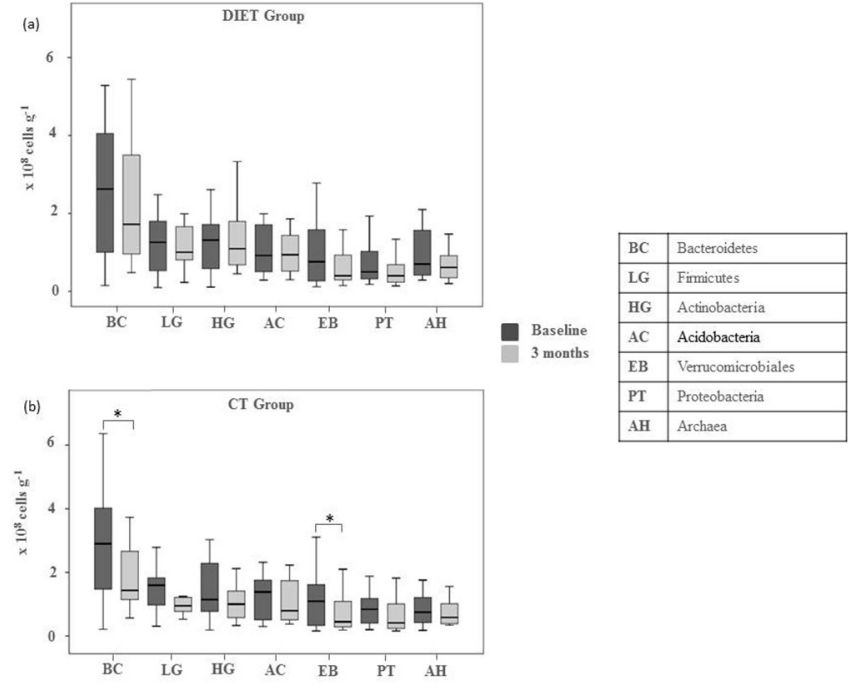

Fig. 3. Characterization of fecal microbiota (domain, phylum and order) of patients with non-alcoholic steatohepatitis at baseline and 3 months. (a), DIET group; (b), CT group. Values described in median and interquartile range, represented by boxplots. ${ }^{\star}$ Statistical difference $(\mathrm{p}<0.05)$ within the group compared to baseline, using Wilcoxon test.

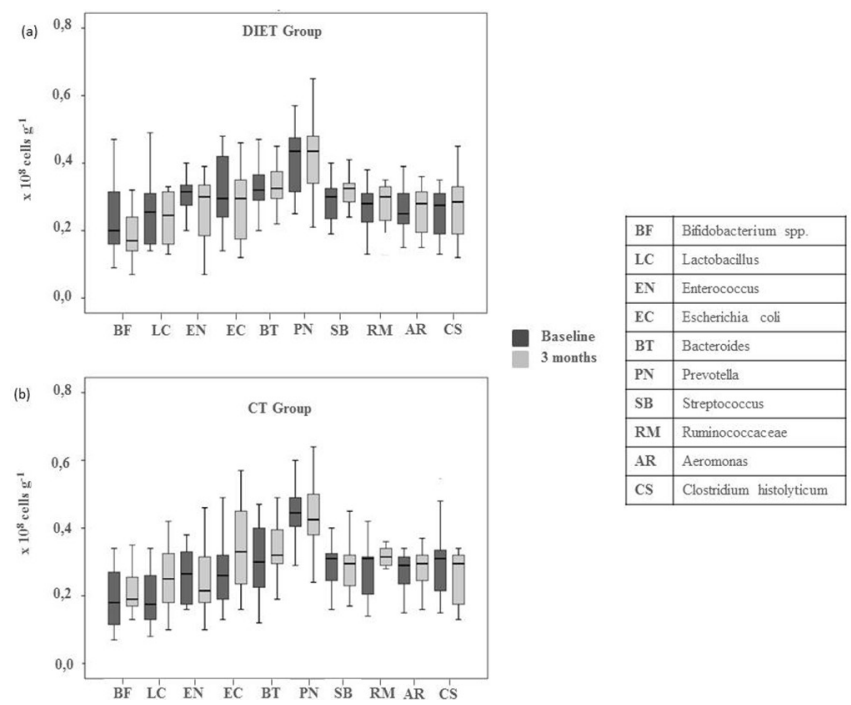

Fig. 4. Characterization of fecal microbiota (gender and species) of patients with non-alcoholic steatohepatitis at baseline and 3 months. (a), DIET group; (b), CT group. Values described in median and interquartile range, represented by boxplots. ${ }^{\star}$ Statistical difference $(\mathrm{p}<0.05)$ within the group compared to baseline, using Wilcoxon test.

\section{DISCUSSION}

The gut microbiota emerges as a potential therapeutic target in the fight against metabolic diseases, including NASH. To the best of our knowledge, this study is the first to investigate the effects of exclusively dietary intervention on the gut microbiota and metabolic-nutritional profile of NASH outpatients. Our hypothesis was that the intervention with individualized prescribed diet combined with nutritional orientation could positively affect all these parameters, when compared to the intervention with only nutritional orientation. 
Table IV. Metabolic-nutritional profile and level of physical activity of patients with non-alcoholic steatohepatitis at baseline and after three months of dietary intervention

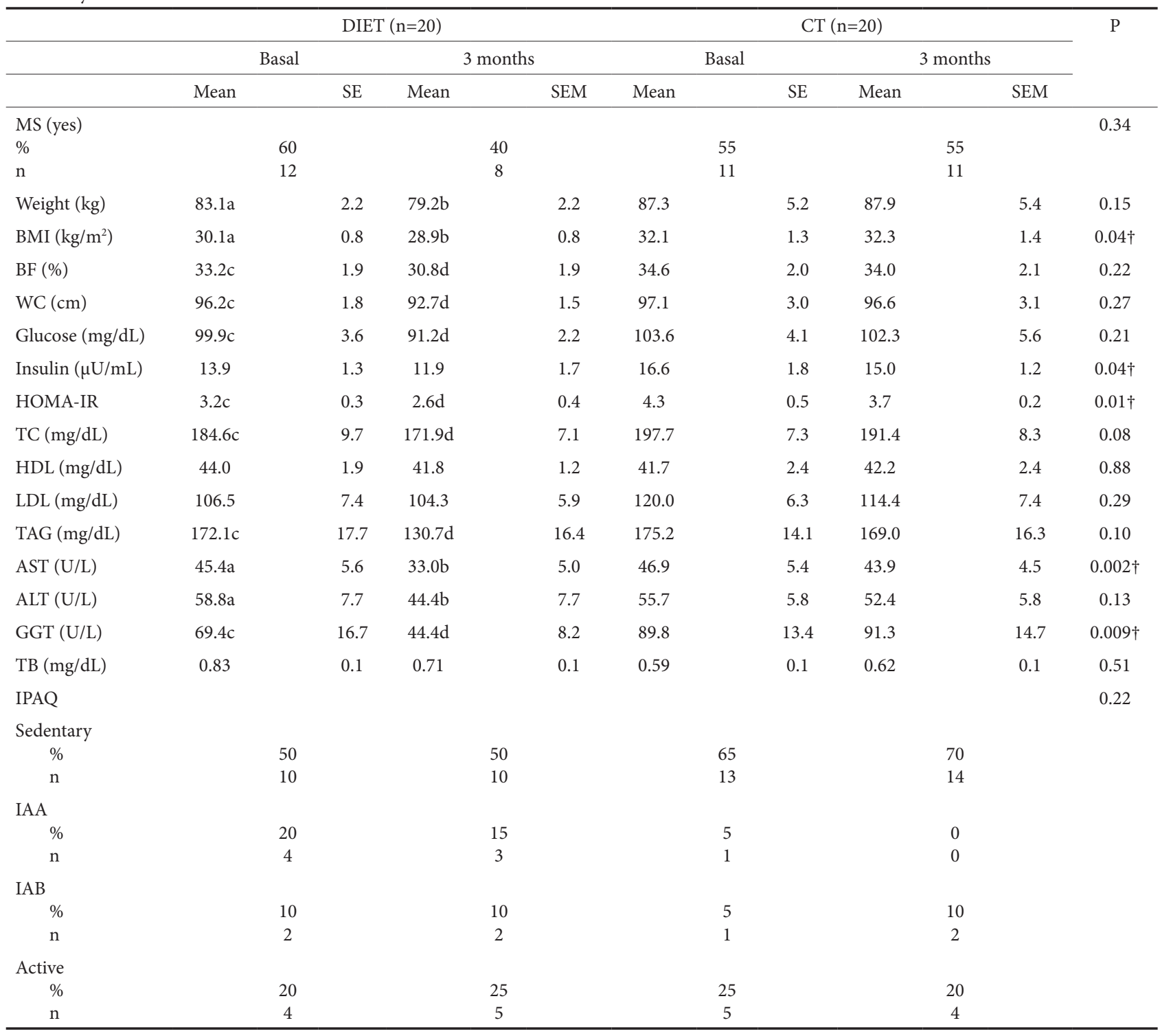

ALT: alanine aminotransferase; AST: aspartate aminotransferase; BMI: body mass index; BF: body fat; GGT: $\gamma$-glutamyltransferase; HDL: high density lipoprotein, HOMAR-IR: homeostasis model assessment of insulin resistance; IAA: irregularly active A; IAB: irregularly active B; IPAQ: International Physical Activity Questionnaire; LDL: low density lipoprotein; MS: metabolic syndrome; TAG: triacylglycerol; TB: total bilirubin; TC: total cholesterol; WC: waist circumference. Data are represented as mean and standard error; a,b Different letters on the same line indicate statistical difference $(\mathrm{p}<0.05)$ within the groups $(t$-test for repeated samples or Wilcoxon); $c, d$ Different letters on the same line indicate statistical difference ( $<<0.001)$ within the groups ( $t$-test for repeated samples or Wilcoxon)

There is evidence that exclusively dietary modification results in positive effects on the gut microbiota of individuals with obesity and/or metabolic syndrome $[18,35]$. In obesity, the consumption of hypocaloric diet rich in fiber, for a month, increased Bacteroidetes and reduced Firmicutes [35]. In our study, we believe that the individualized diet positively affected the gut microbiota of NASH outpatients, since the significant differences observed in the fecal microbiota in the CT group were not repeated in the DIET group. Indeed, the CT group tended to increase potentially pathogenic bacteria, such as Escherichia coli, and to decrease potentially beneficial bacteria, such as Actinobacteria. It is clear in the literature that the genus Escherichia is able to produce ethanol [36], which is associated with inflammation and hepatic injury [37]. On the other hand, Actinobacteria is a phylum of gram-positive bacteria, including the family Bifidobacteriaceae, with protective function during hepatic injury [38]. In addition, the densities of Bacteroidetes and Verrucomicrobiales decreased in the CT group. Some studies have shown an inverse correlation between these microbial groups and obesity-associated comorbidities [39, 40]. Besides that, in the DIET group, there was an increase in the density of total microorganisms, which may reflect in part a greater microbial diversity (greater species richness and uniformity in the distribution of residues among species), considering that the oligonucleotide probes identified at the baseline $73.2 \%$ of the total microorganisms in the fecal samples and $55.5 \%$ of them after the intervention ( $\mathrm{p}=0.001)$. Therefore, future studies should consider the analysis of other bacteria not investigated by this study.

Regarding the SIBO frequency, we observed that dietary intervention in both groups did not significantly modify the percentage of positive tests. However, we observed in clinical 
practice that there was an increase of $80 \%$ of positive tests in the CT group, while the proportion in the DIET group was maintained with the intervention. It is possible that the follow-up of patients for more than three months may show statistical significance among these proportions. SIBO has been associated with increased intestinal permeability and elevated levels of LPS, supporting the role of endotoxemia in the development of steatohepatitis [41]. Considering the possible relationship between SIBO and NASH, therapeutic measures are necessary to prevent or treat gut microbiota imbalance.

Traditionally SIBO treatment consists of eradication of bacteria with broad-spectrum antibiotics [42]. However, the use of antibiotics is not free of risk (e.g., severe adverse reactions, antibiotic resistance and potential for Clostridium difficile infection) [43]. In addition, other treatment options, such as the use of probiotics and modification of dietary habits are under investigation. The modification of dietary habits seems to reduce the frequency of SIBO [44]; however, studies are required to clarify the effects of dietary intervention on the gut microbiota. For example, in irritable bowel syndrome, the use of restricted diet in fermentable oligo-di-monosaccharides and polyols (FODMAPs) normalized the respiratory test in $80 \%$ of SIBO patients [44]. It should be noted that the present study did not standardize a specific type of diet, such as the restricted diet in FODMAPs. We investigated the effects of exclusively dietary modification, adjusted to the individuality of each subject, for the control of potential risk factors for the development and progression of NASH, particularly the gut microbiota.

Although the gut microbiota was the main outcome, we also evaluated the impact of dietary intervention on the metabolic and nutritional profile. We did not find in the literature clinical studies investigating the effects of exclusively dietary modification on these parameters in NASH outpatients. The evidence associated and/or compared diet with physical exercise [45], probiotic [16] or symbiotic [46]. In this way, our work is also the first to conduct this type of analysis.

There is a consensus that lifestyle modification, including strategies for reducing body weight, represents the first line of treatment for NAFLD [47]. Considering the close relationship between obesity and liver disease, body weight should be reduced by at least 3-5\% [48], and a loss of at least 7-10\% may be necessary to improve necroinflammation and liver enzymes [45]. It is worth noting that the evidence for these recommendations has obtained the results after 12 months of follow-up and combined diet with exercise [45]. Our study found a reduction of approximately $5 \%$ of the initial weight in only 3 months of dietary intervention, with no change in the level of physical activity. It is possible that we achieved histological improvement, since the DIET group reduced the serum levels of AST and ALT by more than $20 \%$ and normalized GGT levels. That is, the diet controlled important markers of liver injury, which in NASH have already been correlated with the degree of inflammation and fibrosis [49]. Another relevant finding in the DIET group was the improvement of glycemic and lipid parameters. The DIET group reduced total cholesterol, TAG, glycemia, insulin and HOMA-IR when compared to the CT group, which did not modify any laboratory parameter. Similar results were observed by Elias et al. [50] in patients with NAFLD (including simple steatosis and NASH).

Thus, our results suggest that dietary modifications may contribute to health promotion in NASH outpatients and they should form the basis of nutritional treatment for this condition. Both the DIET and CT groups received the same nutritional orientation, were followed up during the same period by the same nutritionist, and also presented clinical characteristics, dietary intake and similar gut microbiota at baseline. Therefore, we believe that the individualized diet was the factor that promoted positive effects on the gut microbiota and the metabolic-nutritional profile of NASH outpatients.

One limitation of our study was the failure to perform a hepatic biopsy after treatment; however, for ethical reasons, we did not repeat this procedure after dietary intervention to assess liver histology. In addition, we did not evaluate the food consumption of the groups after three months of intervention, which could help in the interpretation of our results; without a measurement of diet adherence, the effect might be a random one. Another limitation was the per protocol analysis rather than intention-to-treat analysis, which preserves the benefit of randomization. Patient follow-up losses during the study may affect the findings, as their unknown response to treatment could change the results of the comparison. However, our study showed a $5 \%$ loss in each group, which does not significantly compromise the results. Finally, we did not exclude potential confounders, such as the presence of diabetes; however, the groups were similar in relation to the percentage of diabetic patients, guaranteeing the homogeneity between them. Future large-scale intervention studies are required to investigate the effects of diet on gut microbiota and metabolic-nutritional profile of outpatients with NASH.

The important strengths of the present study were the design of randomization, the inclusion of only outpatients with NASH diagnosed by hepatic biopsy and the individualization of dietary prescription for outpatients in the experimental group. All of these forces are relevant in comparison to other clinical trials that evaluated the effect of diet in combination with probiotics, symbiotics, and physical exercise for the treatment of NASH $[16,46,45]$.

\section{CONCLUSIONS}

This clinical trial demonstrated that diet plus nutritional orientation improved the metabolic-nutritional profile and resulted in positive effects on the gut microbiota in NASH outpatients, when compared to the nutrition orientation only. The results support the importance of the nutritionist in the multidisciplinary team for the treatment of NASH, individualizing the dietary prescription according to the nutritional needs, preferences and lifestyle of the patient.

\section{Conflicts of interest: None to declare.}

Authors' contributions: F.F.G. designed the concept of the study, and all authors were involved in the literature search and review. F.F.G. wrote the manuscript. D.G.O. performed statistical analysis. A.P.B.M., J.M.O., D.E.C. and L.E.V.V.C.F. were involved in editing the manuscript. All authors read and approved the final manuscript. 
Acknowledgments: The authors gratefully acknowledge the support by the Brazilian Government Organization (Fundação de Amparo à Pesquisa do Estado de Minas Gerais-FAPEMIG; APQ 01522-15).

Supplementary material: To access the supplementary material visit the online version of the J Gastrointestin Liver Dis at http://dx.doi. org/ 10.15403/jgld-197

\section{REFERENCES}

1. Stefan N, Häring HU, Cusi K. Non-alcoholic fatty liver disease: causes, diagnosis, cardiometabolic consequences, and treatment strategies. Lancet Diabetes Endocrinol 2019;7:313-324. doi:10.1016/S2213. 8587(18)30154-2

2. Wanless IR, Lentz JS. Fatty liver hepatitis (steatohepatitis) and obesity: an autopsy study with analysis of risk factors. Hepatology 1990; 12: 1106-1110. doi:10.1002/hep.1840120505

3. Browning JD, Szczepaniak LS, Dobbins R, et al. Prevalence of hepatic steatosis in an urban population in the United States: impact of ethnicity. Hepatology 2004;40:1387-1395. doi:10.1002/hep.20466

4. Losekann A, Weston AC, de Mattos AA, et al. Non-Alcoholic Steatohepatitis (NASH): Risk Factors in Morbidly Obese Patients. In J Mol Sci 2015;16:25552-25559. doi:10.3390/ijms161025552

5. Ekstedt M, Franzén LE, Mathiesen UL, et al. Long-term follow-up of patients with NAFLD and elevated liver enzymes. Hepatology 2006;44:865-873. doi:10.1002/hep.21327

6. White DL, Kanwal F, El-Serag HB. Association between nonalcoholic fatty liver disease and risk for hepatocellular cancer, based on systematic review. Clin Gastroenterol Hepatol 2012;10:1342-1359. doi:10.1016/j. cgh.2012.10.001

7. Bäckhed F, Ding $H$, Wang $T$, et al. The gut microbiota as an environmental factor that regulates fat storage. Proc Natl Acad Sci U S A 2004;101:15718-15723. doi:10.1073/pnas.0407076101

8. Jumpertz R, Le DS, Turnbaugh PJ, et al. Energy-balance studies reveal associations between gut microbes, caloric load, and nutrient absorption in humans. Am J Clin Nutr 2011;94:58-65. doi:10.3945/ajcn.110.010132

9. de Faria Ghetti F, Oliveira DG, de Oliveira JM, de Castro Ferreira LEVV, Cesar DE, Moreira APB. Influence of gut microbiota on the development and progression of nonalcoholic steatohepatitis. Eur J Nutr 2018;57:861-876. doi:10.1007/s00394-017-1524-x

10. Cani PD, Bibiloni R, Knauf C, et al. Changes in Gut Microbiota Control Metabolic Endotoxemia-Induced Inflammation in High-Fat DietInduced Obesity and Diabetes in Mice. Diabetes 2008;57:1470-1481. doi:10.2337/db07-1403

11. Spencer MD, Hamp TJ, Reid RW, Fischer LM, Zeisel SH, Fodor AA. Association between composition of the human gastrointestinal microbiome and development of fatty liver with choline deficiency. Gastroenterology 2011;140:976-986. doi:10.1053/j.gastro.2010.11.049

12. Buzzett E, Pinzani M, Tsochatzis EA. The multiple-hit pathogenesis of nonalcoholic fatty liver disease (NAFLD). Metabolism 2016;65:1038 1048. doi:10.1016/j.metabol.2015.12.012

13. Zhu L, Baker SS, Gill C, et al. Characterization of gut microbiomes in nonalcoholic steatohepatitis (NASH) patients:a connection between endogenous alcohol and NASH. Hepatology 2013;57:601-609. doi:10.1002/hep.26093

14. Wigg AJ, Roberts-Thomson IC, Dymock RB, McCarthy PJ, Grose $\mathrm{RH}$, Cummins AG. The role of small intestinal bacterial overgrowth, intestinal permeability, endotoxaemia, and tumor necrosis factor á in the pathogenesis of nonalcoholic steatohepatitis. Gut 2001;48:206-211. doi:10.1136/gut.48.2.206

15. Shanab AA, Scully P, Crosbie O, et al. Small intestinal bacterial overgrowth in nonalcoholic steatohepatitis:association with toll-like receptor 4 expression and plasma levels of interleukin 8. Dig Dis Sci 2011;56:1524-1534. doi:10.1007/s10620-010-1447-3

16. Wong VW, Tse CH, Lam TT, et al. Molecular characterization of the fecal microbiota in patients with nonalcoholic steatohepatitis - a longitudinal study. PLoS One 2013;8:e62885. doi:10.1371/journal.pone.0062885

17. Del Chierico F, Nobili V, Vernocchi P, et al. Gut microbiota profiling of pediatric nonalcoholic fatty liver disease and obese patients unveiled by na integrated meta-omics-based approach. Hepatology 2017;65:451464. doi:10.1002/hep.28572

18. Haro C, García-Carpintero S, Alcala-Diaz JF, et al. The gut microbial community in metabolic syndrome patients is modified by diet. J Nutr Biochem 2016;27:27-31. doi:10.1016/j.jnutbio.2015.08.011

19. De Oliveira DG, de Faria Ghetti F, de Castro Ferreira LEVV. Association between dietary total antioxidant capacity and hepatocellular ballooning in nonalcoholic steatohepatitis: a cross-sectional study. Eur J Nutr 2019;58:2263-2270. doi:10.1007/s00394-018-1776-0

20. Pocock SJ. Clinical Trials: a practical approach. John Wiley \& Sons, Chichester - New York, Brisbane, Toronto, Singapore; 1983.

21. Matsudo SM, Matsudo VR, Araújo T, et al. Nível de atividade física da população do Estado de São Paulo: análise de acordo com o gênero, idade, nível socioeconômico, distribuição geográfica e de conhecimento. Rev Bras Ciên Mov 2002;10:41-50.

22. Alberti KG, Eckel RH, Grundy SM, et al. Harmonizing the metabolic syndrome: a joint interim statement of the International Diabetes Federation Task Force on Epidemiology and Prevention; National Heart, Lung, and Blood Institute; American Heart Association; World Heart Federation; International Atherosclerosis Society; and International Association for the Study of Obesity. Circulation 2009;20:1640-1645. doi:10.1161/CIRCULATIONAHA.109.192644

23. Kleiner DE, Brunt EM, Van Natta M, et al. Design and validation of a histological scoring system for nonalcoholic fatty liver disease. Hepatology 2005;41:1313-1321. doi:10.1002/hep.20701

24. Friedewald WT, Levy RI, Fredrickson DS. Estimation of the concentration of low-density lipoprotein cholesterol in plasma, without use of the preparative ultracentrifuge. Clin Chem 1972;18:499-502.

25. Matthews DR, Hosker JP, Rudenski AS, Naylor BA, Treacher DF, Turner RC. Homeostasis model assessment:insulin resistance and beta-cell function from fasting plasma glucose and insulin concentrations in man. Diabetologia 1985;28:412-419. doi:10.1007/bf00280883

26. Department of Nutrition for Health and Development, WHO. WHO Global Database on Body Mass Index (BMI): An Interactive Surveillance Tool for Monitoring Nutrition Transition. Public Health Nutrition 2006;9:658-660.

27. Kyle UG, Bosaeus I, De Lorenzo AD, et al. Bioelectrical impedance analysis-part I: review of principles and methods. Clin Nutr 2004;23:1226-1243. doi:10.1016/j.clnu.2004.06.004

28. Ribeiro AC, Sávio KEO, Rodrigues MLCF, Santos CAST, Marchioni DML, Barreto ML. Validação de um questionário de freqüência de consumo alimentar para população adulta. Rev Nutr 2006;19:553-562. doi:10.1590/S1415-52732006000500003

29. Medeiros JD, Araújo LX, da Silva VL, et al. Characterization of the microbial community in a lotic environment to assess the effect of pollution on nitrifying and potentially pathogenic bacteria. Braz J Biol 2014;74:612-622. doi:10.1590/1519-6984.26712 
30. Rezaie A, Pimentel M, Rao SS. How to test and treat small intestinal bacterial overgrowth: an evidence-based approach. Curr Gastroenterol Rep 2016;18:8. doi:10.1007/s11894-015-0482-9

31. Institute of Medicine. Dietary reference intakes for energy, carbohydrate, fiber, fat, fatty acids, cholesterol, protein, and amino acids. Washington (DC): National Academy Press, 2005. doi:10.17226/10490

32. Oliveira CP, Sanches PL, De Abreu-Silva EO, Marcadenti A. Nutrition and Physical Activity in Nonalcoholic Fatty Liver Disease. J Diabetes Res 2016;2016:4597246. doi:10.1155/2016/4597246

33. Jensen MD, Ryan DH, Apovian CM, et al. 2013 AHA/ACC/TOS guideline for the management of overweight and obesity in adults: a report of the American College of cardiology/American Heart Association Task Force on Practice Guidelines and The Obesity Society. Circulation 2014;129:S102-S138. doi:10.1161/01.cir.0000437739.71477.ee

34. Brasil. Ministério da Saúde. Secretaria de Atenção à Saúde. Departamento de Atenção Básica. Guia alimentar para a população brasileira / Ministério da Saúde, Secretaria de Atenção à Saúde, Departamento de Atenção Básica. - 2. ed., 1. reimpr. - Brasília: Ministério da Saúde, 2014. 156 p.

35. Kim MS, Hwang SS, Park EJ, Bae JW. Strict vegetarian diet improves the risk factors associated with metabolic diseases by modulating gut microbiota and reducing intestinal inflammation. Environ Microbiol Rep 2013;5:765-775. doi:10.1111/1758-2229.12079

36. Weimer PJ, Zeikus JG. Fermentation of cellulose and cellobiose by Clostridium thermocellum in the absence of Methanobacterium thermoautotrophicum. Appl Environ Microbiol 1977;33:289-297.

37. Cope K, Risby T, Diehl AM. Increased gastrointestinal ethanol production in obese mice: implications for fatty liver disease pathogenesis. Gastroenterology 2000;119:1340-1347. doi:10.1053/ gast.2000.19267

38. Xing HC, Li LJ, Xu KJ, et al. Protective role of supplement with foreign Bifidobacterium and Lactobacillus in experimental hepatic ischemia-reperfusion injury. J Gastroenterol Hepatol 2006;21:647-656. doi:10.1111/j.1440-1746.2006.04306.x

39. Ley RE, Turnbaugh PJ, Klein S, Gordon JI. Microbial ecology: human gut microbes associated with obesity. Nature 2006;444:1022-1023. doi:10.1038/4441022a

40. Porras D, Nistal E, Martínez-Flórez S, et al. Functional Interactions between Gut Microbiota Transplantation, Quercetin, and High-Fat Diet Determine Non-Alcoholic Fatty Liver Disease Development in
Germ-Free Mice. Mol Nutr Food Res 2019;63:e1800930. doi:10.1002/ mnfr.201800930

41. Ruiz AG, Casafont F, Crespo J, et al. Lipopolysaccharide binding protein plasma levels in liver TNF-alpha gene expression. in obese patients:evidence for the potential role of endotoxin in the pathogenesis of nonalcoholic steatohepatitis. Obes Surg 2007;17:1374-1380. doi:10.1007/s11695-007-9243-7

42. Ponziani FR, Gerardi V, Gasbarrini A. Diagnosis and treatment of small intestinal bacterial overgrowth. Expert Rev Gastroenterol Hepatol 2016;10:215-227. doi:10.1586/17474124.2016.1110017

43. Adike A, DiBaise JK. Small Intestinal Bacterial Overgrowth Nutritional Implications, Diagnosis, and Management. Gastroenterol Clin N Am 2018;47:193-208. doi:10.1016/j.gtc.2017.09.008

44. Pimentel M, Constantino T, Kong Y, Bajwa M, Rezaei A, Park S. A 14-day elemental diet is highly effective in normalizing the lactulose breath test. Dig Dis Sci 2004;49:73-77. doi:10.1023/B:DDAS.0000011605.43979.e1

45. Vilar-Gomez E, Martinez-Perez Y, Calzadilla-Bertot L, et al. Weight Loss via lifestyle modification significantly reduces features of nonalcoholic steatohepatitis. Gastroenterology 2015;149:367-378. doi:10.1053/j. gastro.2015.04.005

46. Ferolla SM, Couto CA, Costa-Silva L, et al. Beneficial Effect of Synbiotic Supplementation on Hepatic Steatosis and Anthropometric Parameters, But Not on Gut Permeability in a Population with Nonalcoholic Steatohepatitis. Nutrients 2016;8:397. doi:10.3390/nu8070397

47. European Association for the Study of the Liver (EASL); European Association for the Study of Diabetes (EASD); European Association for the Study of Obesity (EASO). EASL-EASD-EASO Clinical Practice Guidelines for the management of non-alcoholic fatty liver disease. J Hepatol 2016;64:1388-1402. doi:10.1016/j.jhep.2015.11.004

48. Lu FB, Hu ED, Xu LM, et al. The relationship between obesity and the severity of non-alcoholic fatty liver disease: systematic review and meta-analysis. Expert Rev Gastroenterol Hepatol 2018;12:491-502. do i:10.1080/17474124.2018.1460202

49. Suzuki A, Lymp J, St Sauver J, Angulo P, Lindor K. Values and limitations of serum aminotransferases in clinical trials of nonalcoholic steatohepatitis. Liver Int 2006;26:1209-1216. doi:10.1111/j.14783231.2006.01362.x

50. Elias MC, Parise ER, de Carvalho L, Szejnfeld D, Netto JP. Effect of 6-month nutritional intervention on non-alcoholic fatty liver disease. Nutrition 2010;26:1094-1099. doi:10.1016/j.nut.2009.09.001 


\section{Supplementary material}

Table I. Oligonucleotide probes used for microbial quantification by fluorescence in situ hybridization

\begin{tabular}{|c|c|c|c|c|c|}
\hline Probe & $\begin{array}{c}\text { Target microbial } \\
\text { group }\end{array}$ & Sequence $\left(5^{\prime}-3^{\prime}\right)$ & FA* & $\mathrm{NaCl} \dagger$ & Reference \\
\hline ARCH 915 & Archaea domain & GTGCTCCCCCGCCAATTCCT & 20 & 225 & 1 \\
\hline LGC $354 \mathrm{~A}$ & Firmicutes phylum & TGGAAGATTCCCTACTGC & & & \\
\hline LGC 354 B & & CGGAAGATTCCCTACTGC & 35 & 80 & 2 \\
\hline LGC $354 \mathrm{C}$ & & CCGAAGATTCCCTACTGC & & & \\
\hline HCG 236 & Actinobacteria phylum & AACAAGCTGATAGGCCGC & 30 & 112 & 3 \\
\hline ACIDO 228 & Acidobacteria phylum & TAATCDGCCGCGAMCYCCT & 35 & 80 & 4 \\
\hline ALF 968 & Alpha-proteobacteria class & GGTAAGGTTCTGCGTTT & 30 & 112 & 5 \\
\hline BET 42 a & Beta-proteobacteria class & GCCTTCCCACTTCGTTT & 30 & 112 & 6 \\
\hline GAM $42 \mathrm{a}$ & Gamma-proteobacteria class & GCCTTCCACATCGTTT & 30 & 112 & 6 \\
\hline EPSY 549 & Epsilon-proteobacteria class & CAGTGATTCCGAGTAACG & 30 & 112 & 7 \\
\hline EUB 338 III & $\begin{array}{l}\text { Verrucomicrobiales order } \\
\text { Bacteroidetes: }\end{array}$ & GCTGCCACCCGTAGGTGT & 30 & 112 & 8 \\
\hline BAC 303 & $\begin{array}{l}\text { The most Bacteroidaceae, } \\
\text { some Prevotellaceae, some } \\
\text { Porphyromonadaceae }\end{array}$ & CCAATGTGGGGGACC & 40 & 56 & 9 \\
\hline CF 319 a & $\begin{array}{l}\text { The most Flavobacteria, } \\
\text { some Bacteroidetes, } \\
\text { someSphingobacteria }\end{array}$ & TGGTCCGTGTCTCAGTAC & 35 & 80 & 9 \\
\hline $\begin{array}{l}\text { MUT } 590 \\
\text { SOB } 174\end{array}$ & $\begin{array}{l}\text { Streptococcus mutans } \\
\text { Streptococcus sobrinus }\end{array}$ & $\begin{array}{l}\text { ACTCCAGACTTTCCTGAC } \\
\text { TTAACTCCTCTTATGCGG }\end{array}$ & 30 & 112 & 10 \\
\hline Aero 2 & Some Aeromonas & GTAACGTCACAGCCAGCAGA & 35 & 80 & 11 \\
\hline RUMs 278 & Ruminococcaceae & GTCCGGCTACCGATCGCG & 20 & 225 & 12 \\
\hline Chis 150 & $\begin{array}{l}\text { The most Clostridium } \\
\text { histolyticum (Clostridium } \\
\text { cluster I e II) }\end{array}$ & TTATGCGGTATTAATCTYCCTTT & 30 & 112 & 13 \\
\hline $\begin{array}{l}\text { Pint } 649 \\
\text { Pnig } 657\end{array}$ & $\begin{array}{l}\text { Prevotella intermedia } \\
\text { Prevotella nigrescens }\end{array}$ & $\begin{array}{l}\text { GCCGCCRCTGAASTCAAGCC } \\
\text { TCCGCCTGCGCTGCGTGTA }\end{array}$ & 40 & 56 & 14 \\
\hline Bif 164 & Bifidobacterium spp. & CATCCGGCATTACCACCC & 20 & 225 & 15 \\
\hline
\end{tabular}




\begin{tabular}{|c|c|c|c|c|c|}
\hline Lacto 39 & Lactobacillus & TCTGTTAGTTCCGCTCGCTC & 30 & 112 & 16 \\
\hline \multicolumn{6}{|l|}{ Lacto 15} \\
\hline Enc 1259 & Enterococcus spp. & GAAGTCGCGAGGCTAAGC & 35 & 80 & 17 \\
\hline Enter 2 & Enterococcus & TCCATCAGCGACACCCGAAA & 35 & 80 & 16 \\
\hline Efs 129 & Enterococcus faecalis & ССCTCTGATGGGTAGGTT & 35 & 80 & 17 \\
\hline Eco 1167 & $\begin{array}{l}\text { Escherichia coli } \\
\text { Bacteroides: }\end{array}$ & GCATAAGCGTCGCTGCCG & 40 & 56 & 4 \\
\hline $\mathrm{B} / \mathrm{TAFO}$ & $\begin{array}{l}\text { Tannerella forsythensis } \\
\text { (Bacteroides forsythus) }\end{array}$ & CGTATCTCATTTTATTCCCCTGTA & 30 & 112 & 18 \\
\hline Bfrag 602 & Bacteroides fragilis group & GAGCCGCAAACTTTCACAA & 30 & 112 & 13 \\
\hline Bfrag 998 & Bacteroides fragilis & GTTTCCACATCATTCCACTG & 30 & 112 & 19 \\
\hline
\end{tabular}

* FA: formamide concentration in hybridization buffer.

†Sodium chloride concentration in washing buffer. 